\title{
Tile Classification Using the CIELAB Color Model
}

\author{
Christos-Nikolaos Anagnostopoulos ${ }^{1}$, Athanassios Koutsonas ${ }^{2}$, \\ Ioannis Anagnostopoulos ${ }^{3}$, Vassily Loumos², and Eleftherios Kayafas ${ }^{2}$ \\ ${ }^{1}$ University of the Aegean, Cultural Technology \& Communication Dpt., \\ Mytilene, Greece, GR 81100 \\ canag@ct.aegean.gr \\ http: / /www.aegean.gr/culturaltec/canagnostopoulos \\ ${ }^{2}$ National Technical University of Athens, Electrical \& Computer Engineering School, \\ Athens, Greece, GR 15773 \\ \{loumos, kayafas\} @cs.ntua.gr \\ ${ }^{3}$ University of the Aegean, Information \& Communication System Engineering Dpt., \\ Karlovassi, Samos, GR 83200 \\ janaglaegean.gr
}

\begin{abstract}
An image processing algorithm was developed for tile shade classification on the basis of quantitative measurements in CIELAB color space. A total of 50 tile images of 10 types were recorded, and evaluated with the proposed algorithm in comparison with the conventional classification method. The objectivity of the method is based on the fact that it is not subject to inter- and intra-observer variability arising from human's profile of competency in interpreting subjective and non-quantifiable descriptions.
\end{abstract}

\section{Introduction}

The objective of this paper is the presentation of a method that can be used for the surface inspection and shade classification of ceramic tiles. The shade of a tile is the combination of a number of visual characteristics of the tile surface, including its color and the distribution pattern of color or decoration over the tile surface. Due to the nature of the process, with the use of natural materials, intentionally variable decoration effects and high temperature firing, it is usually not possible to guarantee the production is of a single shade. What is needed is a rational method of establishing a shade classification system for a tile product and then applying it in a consistent way in order to avoid or minimize these problems and so increase profit. Sorting and classification of tiles by shade is a challenging and complicated subject. It is also a critical one for tile manufacturers.

Until now this work is performed almost exclusively by specialized workers, causing several problems to the chain of production. Despite the fact that automated sorting and packing lines have been in existence for a number of years, the complexity of shade classification of tiles has meant that, until recently, automated classification systems have not been possible [1]. However, based on the science of color measurement, attempts are being made to introduce an automatic tile shade classification system [2],[3]. 


\section{Shade Measurements - CIELAB Color Space}

Until now the discussions have been equally applicable to manual shade sorting and automatic shade classification. However, a reliable shade measurement metric should be considered and defined.

Appearance measurement instruments and systems take objective measurements which correspond with these descriptions. The tile surface should be illuminated with one or more light sources of controlled intensity. Then, the light reflected from the tile surface will be acquired, converted to digital signals and sent for further analysis by an appropriate software module.

To be useful for tile shade classification an individual shade measurement should:

- measure an appearance variation that is clearly visible to the human eye, in a way that correlates with human perception.

- give reliable and repeatable, measurement values.

- measure shade variations which occur or could occur during the production of the tile.

- be linear with human perception - a 1 unit difference at one part of the measurement scale should appear to a person to be just as large as a 1 unit difference at another part of the measurement scale.

Moreover, the set of shade measurements selected for tile shade classification of a particular type should:

- be able to detect and measure all of the shade variations that occur or could occur.

- be orthogonal to each other - a change in the appearance characteristic measured by one measurement should not affect any of the other measurements.

- be isotropic - a 1 unit difference of one of the measurements should be perceived as being just as large as 1 unit difference of any of the other measurements.

The work in science of color measurement has been directed at finding ways of measuring and specifying color and color variations. The progress in this field resulted in several well documented and understood color measurement system, defined in international standards under the name of CIELAB.

\section{Algorithmic Description}

This part of the paper constitutes the presentation of the complete software algorithm that will be used for the estimation of shade variation in tile surface.

\subsection{Transformation from RGB to CIELAB}

For the development of such application, it is essential to use a color appearance chromatic model such as the CIE $\mathrm{L}^{*} \mathrm{a} * \mathrm{~b} *$. Consequently, the first, and relatively independent part of application, is the implementation of formulas that make possible the transformation in the CIELAB color space. For fluorescent light the D65 model values were used [5]. 


\subsection{Cluster Segmentation of the Reference Image Using K-Means}

Clustering is a way to separate groups of objects. K-means clustering treats each object as having a location in space. It finds partitions such that objects within each cluster are as close to each other as possible, and as far from objects in other clusters as possible. K-means clustering requires a number of clusters to be partitioned and a distance metric to quantify how close two objects are to each other. Since from step 1, the color information exists in the ' $a{ }^{*} \mathrm{~b}^{*}$ ' space, the objects are pixels with ' $a{ }^{* '}$ and ' $\mathrm{b}{ }^{*}$ values. For the proposed algorithm K-means was used to cluster the objects into $n$ clusters according the Euclidean distance metric, where $\mathrm{n}$ is the number of basic colors in every type of tile.

Using the above method, the user specifies the number of the basic colors without taking into consideration the effect of luminosity. This step was designed in such a way to handle variations of illumination, watersheds and spots that are presented in tile surface. An example is shown in Figure 1, where a picture in Lab -was segmented in 2 sub-images according the Euclidean distance. The basic colors were orange and beige. An algorithm for partitioning (or clustering) $N$ data points into $K$ disjoint subsets $S_{\mathrm{j}}$ containing $\mathrm{N}_{\mathrm{j}}$ data points so as to minimize the sum-of-squares criterion as shown in Equation 1:

$$
J=\sum_{j=1}^{K} \sum_{n \in S_{j}}\left|x_{n}-\mu_{j}\right|^{2}
$$

where $\mathrm{x}_{\mathrm{n}}$ is a vector representing the $n$th data point and $\mu_{\mathrm{j}}$ is the geometric center of the data points in $S_{j}$. In general, the algorithm does not achieve a global minimum of $J$ over the assignments. In fact, since the algorithm uses discrete assignment rather than a set of continuous parameters, the "minimum" it reaches cannot even be properly called a local minimum. Despite these limitations, the algorithm is used fairly frequently as a result of its ease of implementation.

The algorithm consists of a simple re-estimation procedure as follows. First, the data points are assigned at random to the $K$ sets. Then the center is computed for each set. These two steps are alternated until a stopping criterion is met, i.e., when there is no further change in the assignment of the data points.

The clustering step returns the centers of each segmented color in $a^{*}, b^{*}$ values. Supposing that we have $\mathrm{n}$ basic colors in an image the result of K-means clustering will be the following matrix:

$$
K=\left[\begin{array}{ccc}
\text { color } 1 & a_{1} & b_{1} \\
\text { color } 2 & a_{2} & b_{2} \\
\text { color } 3 & a_{3} & b_{3} \\
\ldots \ldots \ldots & \ldots & \ldots \\
\text { color } n & a_{n} & b_{n}
\end{array}\right]
$$

Where $a_{n}, b_{n}$ are the centers of cluster $n$ in the $a^{*} b^{*}$ space.

\subsection{Calculation of Cluster Luminosity}

In the second procedure for K-means clustering, placement of the $\mathrm{K}$ centers can be done by the following procedure, which is similar to the one proposed in [6]. 
- Place K points into the space represented by the objects that are being clustered. These points represent the reference centers found previously in the step of cluster segmentation. Starting with those values the K-means algorithm needs minimum effort to find the new centers according the Euclidean distance.

- Assign each object to the group that has the closest center.

- When all objects have been assigned, recalculate the positions of the K centers.

- Repeat the steps of cluster segmentation and calculation of luminosity until the centers no longer move. This produces a separation of the objects into groups from which the metric to be minimized can be calculated.

As the centers of the clusters become stable for every tile, the calculation of the mean luminosity $\left(\mathrm{L}^{*}\right)$ of the pixels belonging to each cluster follows. Based on the luminosity values, the tiles will be then classified in comparison to the reference $\mathrm{L}^{*}$ values.

\section{Experimental Results}

Tile samples obtained from a Greek industrial company (FILKERAM/JOHNSON) form the training set of the algorithm. Following shade classification by an expert engineer, each tile was labeled with an identifier code of its corresponding type and the appropriate scale shade from 1 to 15 .

The images of the above tiles were then digitized and stored for later retrieval and processing. The hardware for image acquisition, and digitization was a digital camera SONY DSC-V1 with a resolution of $2592 \times 1944$ pixels. The white balance of the camera was set to fluorescent light, as image acquisition was performed in indoor conditions and stable illumination conditions in the laboratory. The distance between the camera and the tile was set equal to $30 \mathrm{~cm}$ with automatic focusing of the camera.

Image analysis was performed by using specialized software (Matlab 6.0 by MathWorks Inc). Following acquisition of the tile image in TIFF format, a file name was given according its type and the shade classification scale (e.g tile5_12.tif corresponds to tile type 5 and shade scale 12). Measurement data were sent by a DDE (Dynamic Data Exchange) method to be saved in a spreadsheet (Excel ${ }^{\circledR}$ by Microsoft) for analysis.

\subsection{Experimental Example}

A small sub-set of 3 tiles of type 1 was considered for demonstration purposes of the developed algorithm. The data set consists of tile1_1, tile1_4, tile1_8, all belonging to tile type 1 and shade scale 1,4 and 8 respectively, where scale 1 indicates darker shade and scale 15 the lighter.

The image tile1_1 is set as the reference tile image. The algorithmic sequence as well as the results for every step are the following:

a. Transformation from RGB to CIEXYZ.

b. Transformation from CIEXYZ to CIELab.

c. Cluster segmentation of the reference image using K-means 
As shown in Figure 1a, tile type1 has a pattern of 2 basic colors, beige and light orange. Therefore, the K-mean software module was activated for $\mathrm{K}=2$ clusters. This step returns, the centers of the two clusters in terms of $\mathrm{a}^{*}$ and $\mathrm{b}^{*}$ values.

Using a simple AND masking technique, the two clusters are now clearly visible in Figures 1b, 1c. Following the masking technique, the mean luminosity value for every cluster is calculated with Equation 2:

$$
\bar{L}_{c}=\frac{\sum_{i=1}^{2592^{c}} \sum_{j=1}^{1944} L_{c}(i, j)}{N_{c}}
$$

where $\mathrm{N}_{\mathrm{c}}$ equals the number of the pixel that belong to cluster $\mathrm{c}$.

Specifically for the tile type 1 , the values $a^{*}, b^{*}$ for every cluster and their average luminosity $\mathrm{L}^{*}$ are:

$$
K=\left[\begin{array}{lll}
c l 1 & a_{1} & b_{1} \\
c l 2 & a_{2} & b_{2}
\end{array}\right]=\left[\begin{array}{ccc}
\text { beige } & 2.2704 & 21.1612 \\
\text { orange } & 6.3916 & 25.3697
\end{array}\right], \quad L=\left[\begin{array}{cc}
c l 1 & 66.1546 \\
c l 2 & 61.1420
\end{array}\right]=\left[\begin{array}{cc}
\text { beige } & 66.1546 \\
\text { orange } & 61.1420
\end{array}\right]
$$

\section{d. Cluster classification}

The above centers $(2.2704,21.1612)$ and $(6.3916,25.3697)$ are now considered to be the reference centers for further cluster processing of similar tile type, while the pair $(66.1546,61.1420)$ corresponds to the reference luminosity values for shade classification.

As the centers of the two clusters are recalculated for every tile of type 1, the calculation of the mean luminosity for each cluster follows. On the basis of the luminosity values, the tile will be then classified in comparison to the reference values.

As a result, the K matrix of tile1_4 and tile1_8 are:

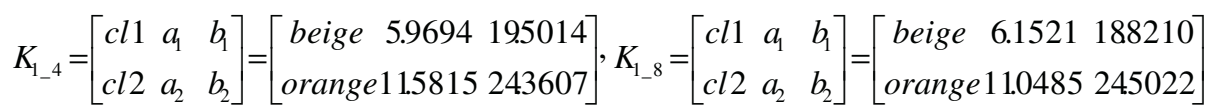

Using the same AND masking technique, the new two clusters for every tile are now depicted in Figures 2b, 2c, 3b and 3c. Using Equation 2 the average luminosity value for each new cluster is given in matrix $\mathrm{L}$ as follows:

$$
L_{1_{-} 4}=\left[\begin{array}{cc}
c 1 & 65.4438 \\
c 2 & 59.2922
\end{array}\right]=\left[\begin{array}{cc}
\text { beige } & 65.4438 \\
\text { orange } & 59.2922
\end{array}\right], L_{1_{-} 8}=\left[\begin{array}{cc}
c 1 & 62.9300 \\
c 2 & 58.4622
\end{array}\right]=\left[\begin{array}{cc}
\text { beige } & 62.9300 \\
\text { orange } & 58.4622
\end{array}\right]
$$

Comparing both $\mathrm{L}_{1-4}$ and $\mathrm{L}_{1-8}$ with the reference matrix $\mathrm{L}$, it is well shown that there is a slight difference in tile illumination values, which is in complete agreement with the experts decision. Therefore, it is assumed that the luminosity measurements in CIELAB space can give a reliable metric for tile classification according their shade, providing that the illumination conditions remain the same for the whole process.

As shown in Figures 1a, 2a and 3a the shade of tile type 1 is the combination of two equally distributed colors over the tile surface. Therefore, no one can safely decide which color plays the most important role for shade classification and it is quite rational 
to consider the average value of both clusters as an adequate shade metric. However, in other tile types the decoration over the surface consists mainly of one color.

Depending on the tile pattern decoration the user may choose which cluster (color) is the most important for luminosity measurements. For instance, for of tile type 1, the manufacturer may consider cluster 1 (beige's) measurement as the most important for shade classification. Consequently, the average luminosity of the basic color should be taken into consideration.

\subsection{Overall Results}

A total of 50 images of 50 individual tiles in various shades belonging to 10 types were recorded. This sample was already classified by conventional classification methods by an expert and labeled accordingly (e.g. tile2_11 for tile type 2 and shade scale 11). Following image processing with the proposed algorithmic procedure those images were classified according the criterion of average luminosity described in step d. Deviations in classification from the expert's decision occurred when the classification metric of average luminosity of cluster 1 and cluster 2 was considered. However, when the average luminosity of both clusters (whole image) is considered to be the classification metric, there is a complete agreement between the algorithmic results and the expert's decision.

\section{Discussion}

An automatic tile inspection system implementing digital cameras and image processors could be used for tile classification. It would be able to calculate shade measurements and exploit these measurements in a trustworthy and repeatable way. It is proved herein, that it is possible to utilize image processing methods in CIELAB color space to build up a reliable shade classification scheme compatible to human color perception. This would possible, however, if the algorithm developer has a clear understanding of the requirements and theory of tile shade classification, and experience of what shade measurements are required for different tile types. Potential applications of such systems include quality control and shade-variation monitoring in the industry of ceramic materials. The benefits introduced are cost reduction and quality improvement as also shown in [7].

\section{References}

1. Vincent LEBRUN,"Quality control of ceramic tiles by machine vision," Flaw Master 3000, Surface Inspection Ltd. 2001.

2. C. Boukouvalas, F. De Natale, G. De Toni, J. Kittler, R. Marik, M. Mirmehdi, M. Petrou, P. Le Roy, R. Salgari and G. Vernazza. An Integrated System for Quality Inspection of Tiles. Int. Conference on Quality Control by Artificial Vision, QCAV 97 , pp 49-54, France, 1997.

3. Murat Deviren, M.Koray Balci, U. Murat Leloglu, Mete Severcan, "A Feature Extraction Method for Marble Classification", Third International Conference on Computer Vision, Pattern Recognition \& Image Processing (CVPRIP 2000), Atlantic City, pp 25-28. (HSI) 
4. A. D. H. Thomas, M. G. Rodd, J. D. Holt, and C. J. Neill. Real-time industrial visual inspection: A review. Real-Time Imaging, 1:139-158, 1995.

5. Mark D. Fairchild, Color Appearance Models, Addison-Wesley, Reading, MA (1998).

6. Park., S.H., Yun, I.D., Lee, S.U., "Color Image Segmentation Based on 3-D Clustering: Morphological Approach”, Pattern Recognition, Vol. 31. No. 8, pp. 1061-1076, 1998.

7. Martin Coulthard, "Tile shade classification strategies for maximum profit", Tile \& Brick international journal, Vol.: 16, $\mathrm{N}^{\circ} .: 3$, June, 2000.

\section{Appendix: Example Screenshots}
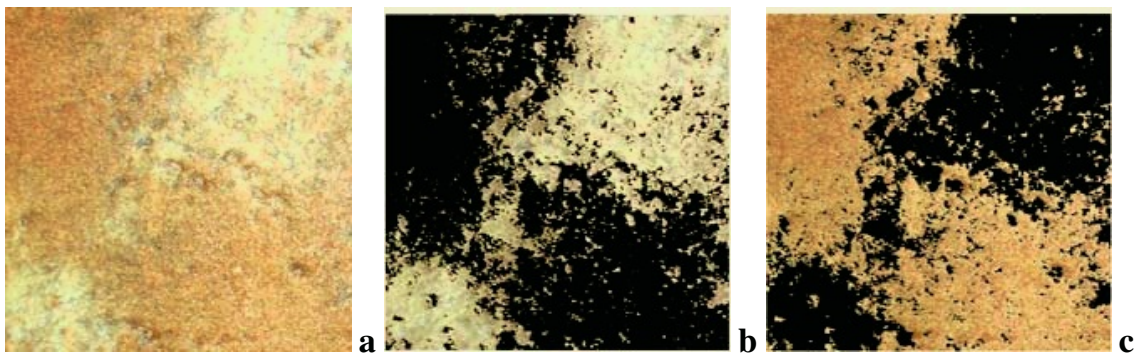

Fig. 1. a) Tile1_1, b) Cluster 1 (beige): $a^{*}=2.2704, b^{*}=21.1612, L^{*}=66.1546$, c) Cluster 2 (orange): $\mathrm{a}^{*}=6.3916, \mathrm{~b}^{*}=25.3697, \mathrm{~L}^{*}=61.1420$
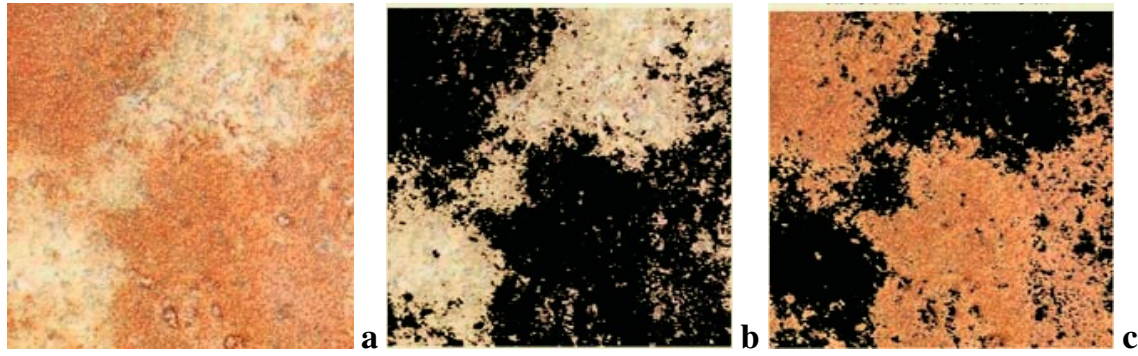

Fig. 2. a) Tile1_4, b) Cluster 1 (beige): $a^{*}=5.9694, b^{*}=19.5014, L^{*}=65.4438$, c) Cluster 2 (orange): $\mathrm{a}^{*}=11.5815, \mathrm{~b}^{*}=24.3607, \mathrm{~L} *=59.2922$
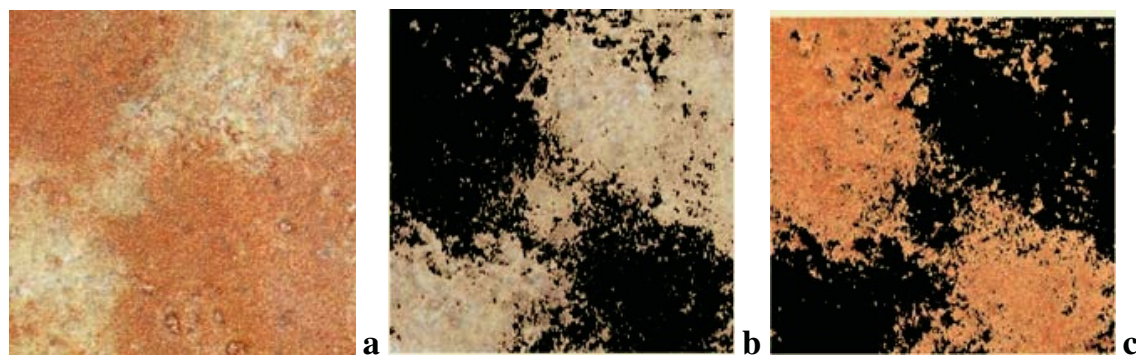

Fig. 3. a) $1 \_8$, b) Cluster 1 (beige): $a^{*}=6.1521, b^{*}=18.8210, L^{*}=62.9300$, c) Cluster 2 (orange): $\mathrm{a}^{*}=11.0485, \mathrm{~b}^{*}=24.5022, \mathrm{~L}^{*}=58.4622$ 


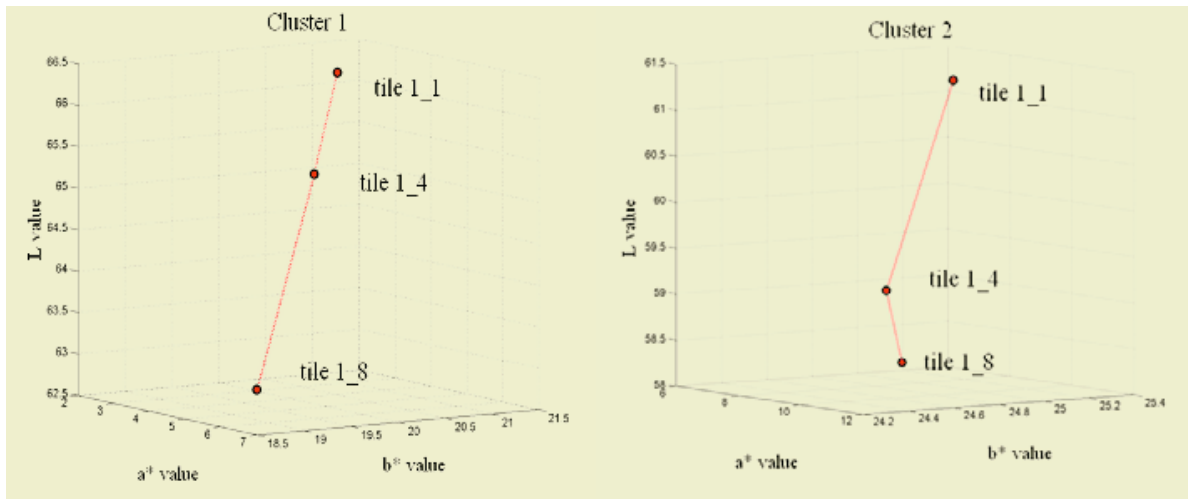

Fig. 4. Visual representation of how values $L^{*}, a^{*}$ and $b^{*}$ are positioned in the CIELAB color space for clusters 1 (beige) and 2 (orange) in tiles 1_1, 1_4 and 1_8 